Voix et Images

volXetimages

\title{
Des fous et des autres
}

\section{André Brochu}

Volume 10, numéro 3, printemps 1985

André Major

URI : https://id.erudit.org/iderudit/200523ar

DOI : https://doi.org/10.7202/200523ar

Aller au sommaire du numéro

\section{Éditeur(s)}

Université du Québec à Montréal

\section{ISSN}

0318-9201 (imprimé)

1705-933X (numérique)

Découvrir la revue

\section{Citer cet article}

Brochu, A. (1985). Des fous et des autres. Voix et Images, 10(3), 179-187.

https://doi.org/10.7202/200523ar d'utilisation que vous pouvez consulter en ligne.

https://apropos.erudit.org/fr/usagers/politique-dutilisation/ 


\title{
Poésie
}

\section{Des fous et des autres}

\author{
par André Brochu, Université de Montréal
}

D'abord, trois livres fous. Le Contenant, de Christine Daffe', commence comme un récit à peu près sage sinon conventionnel, puis vire au cauchemar, aux convulsions, à la démence. On dirait vraiment un discours psychotique, avec toutefois cette part d'invention réelle qui signale la santé (sinon la poésie). Le langage devient alors parfaitement performatif: il crée à mesure ce dont il parle, il impose une situation locutoire où le référent n'a pas d'autre lieu que l'énoncé. Sans doute est-on loin de la littérature, mais peut-être pas de son renouvellement. Le Contenant ne ressemble à rien de ce qu'on connaît, pas même à Gauvreau ou Artaud, et pourrait ouvrir des voies à quelques explorateurs peu sujets au vertige.

Dans l'Oekoumène écorché vif, Michel Janvier² hurle gaîment une souffrance quelque peu éthylique, sous le signe de «l'Overdose» et de «la Luxure». Le délire y est contrôlé, même si l'auteur succombe aux facilités d'usage, qui accréditent une globale exaspération à l'égard des inepties de l'existence et des moyens par lesquels on tente de les oublier: drogue, sexe et autres «conneries». Expressionnisme parfois truculent, parfois un peu gris. Des trouvailles vulgaires et pas bêtes, dans ce style: «au nom du cul / et du vice / et du sein tari / ainsi boit-il.» À lire en contexte, bien entendu. Ce genre de poésie doit tout au déferlement. Le galet qu'on y ramasse, pour en zieuter de plus près la magie, perd illico ses couleurs de l'autre monde.

Il faut parcourir aussi la poésie d'Anonyme Sanregret (un pseudonyme, je parie!), pour l'attentat perpétré contre nos habitudes de lecture. Inutile ${ }^{3}$ est un livre beau, pop, new wave, ce qu'on voudra, et superbement présenté. L'illustration, due à Philippe Brochard, Suzanne Duranceau, Christine Lajeunesse, OBU international et Richard Parent, compte autant que le texte. Le langage du poème rivalise de bêtise (au bon sens du mot!) avec les images de bande dessinée; on croirait un opéra sur papier, qui crie lui aussi - comme l'Oekoumène écorché vif - l'ineptie du quotidien, avec d'épouvantables facilités: «Mes yeux sont des néons / de la réalité urbaine» - ! Il n'empêche: le rapport texte / image est neuf, suggestif, créateur.

Autre réalisation graphique étonnante, et bien davantage encore: Tanguer, de Gilles Lacombe ${ }^{4}$. Sous emboîtage de fort carton, douze planches de $38 \times 57 \mathrm{~cm}$ où l'auteur explore diverses façons de réaliser le calligramme total. Les mots, tantôt lisibles, tantôt pas, s'avouent carrément lignes, formes, matière à dessin. Toute entreprise de déchiffrement est tôt ou tard découragée, le lecteur est remis à sa place de contemplateur, non sans qu'il ait frôlé l'aventure suprême: lire un tableau, regarder le Langage. 


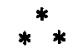

J'arrive maintenant à un livre important, où d'aucuns ont voulu voir un amas d'inepties. Nicole Brossard est adulée des uns, décriée des autres. À chacun ses passions. Le formalisme n'est pas mon pain quotidien mais j'éprouve du respect, de l'admiration et souvent un réel plaisir de lecture devant les textes réunis dans Double Impression 5 . Textes et poèmes intercalaires, si l'on veut, car ils n'ont pas trouvé place dans les nombreux livres qui jalonnent la carrière de l'auteur. Ils sont comme leur après-coup ou leur pré-face, ils sont hors texte et pourtant, ensemble, ils forment ce texte diagonal, pliure de l'oeuvre complet, où se saisit la raison de l'ensemble. Certains, décisifs, tels «Vaseline» et «E muet mutant», manifestes de l'écriture féminine (ou au féminin) où une grande lucidité s'unit à une non moins grande générosité. Je m'étonne qu'on ait parlé de narcissisme, ou encore d'écriture malhabile. Nicole Brossard, au centre de l'aventure poétique des quinze dernières années, dont elle fut l'une des inspirations majeures, est à mes yeux l'image même de l'écrivain faisant oeuvre avec une patience et une sûreté admirables, passionnée et gardant pourtant une parfaite égalité, subordonnant tout à son travail. Double Impression est l'attestation exemplaire de cette oeuvre, de cette continuité.

Une lecture attentive permet deux constatations apparemment contradictoires: 1. La théorie, comme telle, intéresse peu ou pas Nicole Brossard: elle est du côté de la "création", ou mieux de la fiction, même si tout texte qu'elle écrit «s'offre la question sur une écriture qui n'efface pas son origine, qui au contraire la tente et la creuses. La fiction se pose comme effet d'une intention particulière de produire du sens et comme mise en oeuvre contrôlée des moyens langagiers. 2. L'univers de Nicole Brossard, bien que non théorique, est abstrait, peu figuratif (ou représentatif). Le corps, par exemple, y est surtout réduit à sa surface, ses «surfaces de peau»; on songe au moderne refus de la profondeur proclamé par Deleuze. Tout est plans, lignes, organes sans interconnexions.

L'écriture est donc une pratique fort concrète, mais pratique de l'abstrait. L'auteur la présente adéquatement en ces termes, non dénués d'humour: «... car c'est bien connu qu'à travers mes mots, plus de jeu que de pensée, d'exactitude idéologique. Cependant plein d'indices, de sens à élargir, à explorer». Et c'est vrai. Et c'est beau. Pour qui aime l'écriture, et même la poésie. Mais le lyrisme ici n'est plus «fait pour durer comme une vieille couverture grise dans un lycée une édition originale», il est dépense immédiate et intégrale du sens, de tous les sens.

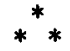

Le dernier recueil de Michel Beaulieu, Kaléidoscope ${ }^{6}$, porte bien son titre si je m'en rapporte au souvenir que m'ont laissé ces jouets, merveilleux 
mais vite décevants, qui ont (comme on dit) enchanté mon enfance. Quelques morceaux de plastique transparent aux couleurs vives, multipliés symétriquement par les miroirs, composaient des bouquets, des étoiles, des feux d'artifice jamais semblables, et pourtant prisonniers d'une maléfique continuité. À peine coloraient-ils l'ennui des heures creuses. On sentait obscurément que cette fragile magie était suspendue aux caprices de l'entropie (le sous-titre du recueil: les Aléas du corps grave).

Ajoutons à cela que les paysages lumineux que Michel Beaulieu nous donne à contempler ne s'écartent guère des tons de gris. Très peu de rêve, ni d'images. Une sorte de prose déviée, soumise au rythme poétique mais qui se souvient trop souvent d'être prose. De triviales anecdotes - par exemple un cardinal, à l'identité transparente, monté à bord du même avion que le poète - des vulgarités de don Juan visqueux - «tu te tais mais ça fait rien / fait-elle en glissant sous les draps / tu donnes un maudit bon coup / de queue quand même» -, des nostalgies d'usager des transports en commun - «le $3 \mathrm{~A}$ jusqu'à Girouard / le 48 jusqu'à Snowdon / le 17 jusqu'à Cartierville...» - agrémentent mal le chant monotone du désir et de la souvenance. La plupart du temps, le poète se parle à lui-même et cette substitution du $t u$ au je, tout en favorisant la narration anecdotique, crée une mise à distance du sujet. Le moi devient ainsi le héros d'un énoncé où triomphent l'égalité métrique, la continuité, lesquelles dissolvent les aspérités du vécu et imposent l'idée d'une gravité des choses, qui pèsent vers la mort. Au bout du compte, très globalement, Kaléidoscope est une réussite, dans une veine qui se situe loin du formalisme comme de l'expressionnisme et qu'on pourrait appeler le référentialisme. Mais la marge qui sépare cette tendance esthétique du pur et simple prosaïsme est bien étroite.

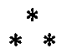

Dans Intérieurs, France Théoret ${ }^{7}$ renouvelle par un traitement sobre et convaincant un vieux lieu commun littéraire, celui du moi (ici le moi est femme) muré en lui-même, privé de voix, démuni au milieu d'un immense abandon. Aucun bavardage, mais le discours n'est cependant pas anémique. Il est dense, tragique et surtout beau, de cette beauté qui transforme l'expression d'un destin individuel en symbole multiple, ouvert aux identifications. On se reconnaît, on se connaît à neuf dans ces traits de plume navrés:

la voix s'est recouverte silence sauvage

la tête entrée dans le réel

le corps laissé seul

au jeu de massacre

Une sorte d'histoire se dessine, celle de l'apprivoisement d'une "vaste demeure délabrée» dont pendent tous les fils, maison «sans murs» qui refuse son abri. Il faut tout refaire, enfouir les renvois, les raccorder à 
l'égout: «Eaux propres et eaux usées. Partage des eaux.» En somme, tout l'ordre vital est à réinventer, tout l'espace domestique, quotidien à aménager pour rendre cette maison, comme soi-même, habitable, c'est-àdire organiquement viable (par référence au «corps sans organes» du schizophrène deleuzien; ici, les organes sont tous là mais débranchés). Et ce n'est jamais fini:

suspension indéfinie des travaux

à l'intérieur cette femme

en transformation corporelle

La maison est le symbole transparent du corps, et l'une et l'autre sont en mutation indéfinie. C'est le thème du transit (de l'attente interminable), que France Théoret développera dans un autre texte 8 .

On se souvient d'Anne Hébert - le Tombeau des rois, le Torrent devant une détresse comme celle-ci, liée à une volonté antérieure: «Des silences obscurs m'ont fait devenir cette femme intérieure au rêve avalé, poursuivie, figure nodale, traversée par la violence passée, endettée par une mémoire commune.» Pieds et poings liés, le poète est cette femme qui interroge la nuit et lui oppose un orgueil dur et beau comme la mort.

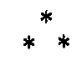

La poésie de Jean Chapdelaine Gagnon, dans Essaime ${ }^{9}$ et Entrailles ${ }^{10}$, n'est pas de celles qu'on peut laisser de côté, bien qu'elle ne s'impose pas sans peine à l'attention du lecteur. Remarquablement écrite, intelligente, fine, cultivée, elle manque peut-être de cette qualité d'émotion qui la rendrait plus immédiatement parlante. On s'émerveillera quand même de ce monde où tout vire au blanc ou à la pulvérulence, où le rire de la femme met un homme en pièces, où les dos d'or, bêtes fabuleuses qui défèquent par la bouche, avalent les hommes par leur ventre ouvert.

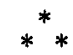

«Comprenez-vous? Comprenez-vous qui je suis si vous êtes? Devant le feu de nous?" La syntaxe de la relation entre les êtres, entre le moi et ses proches, ses amants ou ses amis, fait la matière principale du dernier recueil d'Élise Turcotte, Navires de guerre ${ }^{11}$, au titre trompeur car on n'y trouve rien de bien belliqueux. Le langage est accessible, peu imagé et frôle parfois la banalité: «Ça pourrait être maintenant, dans le long couloir illuminé de soleil, deux corps se pénétrant jusqu'à se défoncer.» La sexualité, l'enfance sont les points d'ancrage d'une poésie du sujet, où abondent les notations de bonheur: "Les petites filles en moi savouraient l'avenir en riant.» Certes, les occasions de tristesse ne manquent pas en ce monde, mais on refuse «que la vie ne soit qu'un rendez-vous manqué». Il faut "encourager le réel d'advenir, immédiatement, avec tous les contours possibles de l'impossible». Cet optimisme, foncièrement intelligent, est une denrée 
savoureuse et bien rare. Le précédent recueil d'Élise Turcotte, Dans le delta de la nuit 12 , contenait déjà cette profession de santé: «J'écris le refus mais la vie sur mon visage.»

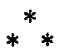

Universitaire de renom, Joseph Bonenfant publie son premier recueil, Grandes Aires ${ }^{13}$, à un stade de sa carrière où beaucoup de ses collègues ont renoncé, pour leur part, à réaliser leurs aspirations littéraires de jeunesse. Le résultat est fort sympathique. L'inévitable maturité du texte n'étouffe pas la vitalité du langage; l'auteur trouve d'heureuses formules pour célébrer la fusion de l'écriture et du corps, ou pour évoquer la position de l'homme avançant dans sa vie et refusant le recours de plus en plus dangereux à l'enfance.

On peut juger un peu restreint l'univers où se confine la pensée du poète. C'est celui des problématiques intellectuelles, Lacan, Deleuze, la modernité, qui sont de «grandes aires» mais où la poésie ne trouve pas toujours l'espace suffisant pour prendre son envol. Mais Bonenfant ne se limite pas aux "fictions théoriques», il sait aussi évoquer le monde actuel, qu'il s'agisse du Quétec («Tunnel pq»), de Montréal («le pire trou de la planète sans ses restaurants, ses fêtes, ses cinémas, ses amours, ses néons, et son grand fleuve stellaire») ou de la fin universelle des croyances. Parole intelligente et chaleureuse, qui reste subordonnée au discours critique - celui de Bonenfant est l'un des plus suggestifs de notre littérature - mais qui a tout de même son autonomie et offre de substantiels plaisirs de lecture.

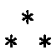

Après deux romans intitulés Dieu et l'Existencel4 - rien de moins! Carole Massé publie l'Autre, qui se présente comme un poème en plusieurs chants brefs, sous forme de prose. Le titre rappelle les précédents par sa couleur métaphysique et l'exceptionnelle ampleur de son référent : comme Dieu, comme l'existence, l'autre est virtuellement toute chose, comprend l'infini moins le moi. Nous sommes en pleine ontologie, et pourtant aux antipodes de l'humanisme et des philosophies traditionnelles, même des philosophies récentes comme celle de Heidegger, chercheuses de vérité. Il s'agit d'une ontologie-fiction, forgée au plus près du vécu, rigoureusement personnelle et à usage privé; il y a comme un détournement des «grandes significations», du sacré que véhicule le langage, en vue d'une affirmation du sujet désirant. Tout se passe comme si la scolastique de l'enfance, décapitée de ses axes symboliques, devenait matière à jeu autour d'un vide essentiel, cette «case 16» du puzzle dont parle Carole Massé dans «La femme à l'écritoire 16 》 - on peut y voir, mutiplié par deux, le chiffre de l'infini. Quel que soit le sujet qu'elle aborde au long de ces quarante pages, l'auteur nous fait toujours entrevoir «les couleurs des choses de tous les temps», le particulier se dissout dans l'universel, suppori du vide, de l'infini 
justement. Le même n'est jamais le même, il «ressemble étrangement à l'autre», et quand il aime, on se demande s'il aime «de l'amour de l'autre qui aime ou l'aime, ou de son propre amour d'aimer ou d'être aimé». Grave question! On souhaiterait bien, cependant, avoir plus de prise sur le texte, diablement hermétique. Le sens se déplace toujours, comme cette case 16 qu'une touchante maladresse fait immanquablement surgir oủ il ne faut pas, venant briser la série au moment où elle allait enfin être constituée.

L'écriture est un jeu, mais s'il est trop compliqué, on risque, hélas! d'en jouer tout seul.

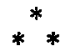

Le grand plaisir de déconcerter: "C'est comme si on cultivait des champs de fantômes et qu'elles passaient à la perfection.» Des fantômes au féminin? «C'est brutal, d'accord, mais le réveil n'arrange rien.» D'accord: vous avez saisi l'astuce? L'accord grammatical, voilà ce dont il est question, voilà ce qui brutalise vos petits réflexes linguistiques. Roger des Roches adore la brutalité. Poème, attention! suivi de Deuxième Poème ${ }^{17}$ est écrit en coup de vent ${ }^{18}$, avec un grand luxe d'humour, de formules saisissantes, de fantaisies érotiques qui, chose rare chez un poète, vous tiennent en haleine. Je ne connais pas de langage plus expressif dans notre poésie depuis Gauvreau. Mais l'expressivité ne fait qu'un avec la lucidité d'une écriture attentive à ses propres effets, et d'un vécu où l'ironie corrige constamment la tendresse. On hésite: tantôt Des Roches apparaît comme un Narcisse souverain, tantôt comme l'éducateur de nos sensibilités, qu'il voudrait épurer de leur bêtise. Masochistes, attention!

Comme tournant la page réunit les poèmes d'Alexis Lefrançois écrits entre 1968 et 1978 et publiés, sauf quelques-uns, en divers recueils ${ }^{19}$. Il s'agit donc d'une rétrospective, qui permet d'apprécier la constante qualité d'une écriture simple, droite, séduisante, formellement irréprochable et malheureusement glaciale, malgré tout ce qu'elle comporte de limpidité et de transparence. On est spontanément conquis par les mots lumineux du poème, les architectures raffinées, délicates, faites de reprises judicieuses, de rythmes savants, de recours calculés aux formes traditionnelles (l'alexandrin); le tout est enchanteur, et pourtant une sorte de refus se profère en filigrane, très bien thématisé du reste dans ces quelques vers:

jeux froids comme nés de la couleur glissements de glacier très haut calmement du blanc sur le blanc glissement

comme immobile figé

sur quatre ou cinq états de moi-même en suspens 
C'est la couleur, paradoxalement, qui génère le froid, prépare le triomphe du blanc, fige les paysages du moi. Une fraîcheur éluardienne prélude aux enchantements du calcaire, à des crispations mallarméennes, comme pour leur prêter la vie dont ils sont dépourvus. Le mot, "comme un laurier penché», conjugue ainsi «la clarté / de turquoise et de flamme» et l'allure d' 'idéogramme blanc sur le vide de l'air». Le laurier, le marbre, qui rappellent l'immortalité poussiéreuse des Anciens, sont la pierre et le végétal préférés, dans une poésie qui tourne le dos au réel et regarde fixement la mort. «Le silence est le terme.»

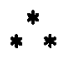

Connu et apprécié pour le travail considérable qu'il accomplit comme secrétaire général de l'Union des écrivains québécois, Jean-Yves Colette s'adonne depuis vingt ans à une rigoureuse entreprise d'écriture. Préliminaires $^{20}$ réunit les textes des cinq premières années, rendus à leur ordre chronologique de composition et livrés dans une édition ne varietur, avec l'appareil critique dont l'auteur, mieux que quiquonque, pouvait les entourer. Il s'agit d'une publication, pour la postérité, de «préliminaires» au cachet de testament; mais le lecteur d'aujourd'hui y verra un livre attrayant, où s'ébauche une oeuvre tout éprise de perfection. Oeuvre étrange, difficile, où le moi semble souvent muré dans l'angoisse d'une mort qui enlève tout sens à son action; où l'incommunicabilité qui en résulte, notamment en amour, est curieusement contournée, sinon démentie, par l'interversion du locuteur; le poète devient la femme aimée (vraiment aimée?), rêve, parle, vit (à) sa place: l'impossible discours avec l'autre devient l'implacable discours de l'autre, disant la mort, disant la vie et leur obscène trivialité. Thème fort général. Un sens ecclésiastique - avec jeu de mots - de la vanité de tout inspire quelque part ce commentaire métatextuel: «Notez bien l'emploi de mots vulgaires et dégradants comme je t'aime et dégueulasse mais cela n'a pas de sens et c'est autrement dit des paroles inutiles.» Le texte non seulement affirme l'égalité de mots comme je t'aime et dégueulasse, tous deux vulgaires au même titre que tout ce qui existe, mais il dénonce sa propre absurdité, disqualifiant le signe après le référent.

Le dégoût métaphysique inspire un formalisme de plus en plus hermétique, ou parfois des textes délibérément niais, gratuits, tels «Les gros coussins mous» et "De la motte de terre à la motte de Gudule» (mais déjà "Le sourire d'un noeud coulant» et "(Sans légende)»). On a alors l'impression d'une inspiration creuse, voulue, celle de quelqu'un qui voudrait se servir de la littérature dans un combat où elle n'a que faire - contre la mort. 
Bon. Je renonce à rendre compte de tout. J'aurais aimé parlé des recueils suivants, tous de 1984 sauf mention contraire, qui méritent une lecture attentive:

- aux éditions des Forges, Sur le fond de l'air de Louis Jacob (60 p.); les Jardins de l'aujourd'hui de Michel Muir (82 p.); Orifices de Denuis Saint-Yves (68 p.); Papier sans bords de Jean-Pierre Leroux (74 p.);

- aux éditions du Vermillon, d'Ottawa, les Sublimes Insuffisances d'André Leduc ( 86 p.);

- aux Presses Laurentiennes, le Choix de Jacqueline Vézina dans l'oeuvre de Medjé Vézina (78 p.);

- aux éditions du Remue-Ménage, les Rendez-vous par correspondance suivi des Prénoms de Louise Cotnoir (100 p.);

- aux éditions de la Pleine Lune, les Coïncidences terrestres de Yolande Villemaire (s.p.);

- à la revue Lèvres urbaines, Droite et de profil (No 7) d'Anne-Marie Alonzo (16 p.) et Jeunes Femmes rouges toujours plus belles (No 8) de Yolande Villemaire ( 24 p.);

- chez VLB éditeur, Rock-désir, chansons de Lucien Francoeur (188 p.) et la Lettre infinie de Madeleine Gagnon (108 p.);

- au Noroît, Euphorismes de Pierre Laberge (56 p.);

- aux Herbes rouges (revue), Aucune Intention de bonheur (No 122) de Guy Moineau (48 p.) et Nuits (No 126) d'André Roy (44 p.);

- à l'Hexagone, Écrire ou la Disparition de Michel Leclerc (52 p.);

- aux éditions Parti pris, Femme, objet du regretté Louis Geoffroy (1983; 90 p.);

- enfin, aux éditions Saint-Germain-des-Prés, Près de Cécile Cloutier de Lantagne (1983; 32 p.).

On voit que la matière ne manque pas, et que le trop rare amateur de poésie est admirablement servi, grâce à une édition artificiellement prolifique, qui bénéficie de subventions considérables. Pour une vue pointilliste mais assez complète et fort chaleureuse de la production poétique des dernières années, on lira les Livres parlent de Claude Beausoleil21, l'un des principaux artisans du renouveau poétique des années 70 et son principal publiciste grâce à ses chroniques dans le supplément littéraire du Devoir. Celles-ci ont permis à de nombreux lecteurs de s'initier à la problématique de la modernité et de franchir la barrière du lisible qui les séparait du poème.

1. Christine Daffe, le Contenant, Montréal, Soudeyns-Donzé éditeurs, 1984, $62 \mathrm{p}$.

2. Michel Janvier, l'Oekoumène écorché vif, Montréal, Parti pris, 1983, 72 p.

3. Anonyme Sanregret, Inutile, Montréal, Voltichenkö, 1984, 60 p.

4. Gilles Lacombe, Tanguer, Ottawa, Éditions de l'Université d'Ottawa, 1984, s.p.

5. Nicole Brossard, Double Impression, poèmes et textes 1967-1984, Montréal, l'Hexagone, $1984,142 \mathrm{p}$. 


\section{CHRONIQUES 187}

6. Michel, Kaleidoscope ou les Aléas du corps grave, Saint-Lambert, le Noroît, 1984, $154 \mathrm{p}$.

7. France Théoret, Intérieurs, Montréal, revue les Herbes rouges, No 125, 1984, 40 p.

8. France Théoret, Transit, Montrèal, revue les Herbes rouges, No 129, 1984, $46 \mathrm{p}$.

9. Jean Chapdelaine Gagnon, Essaime, Saint-Lambert, le Noroît, 1983, 112 p.

10. Jean Chapdelaine Gagnon, Entrailles, Trois-Rivières, Écrits des Forges, 1984, 76 p.

11. Élise Turcotte, Navires de guerre, Trois-Rivières, Écrits des Forges, 1984, 64 p.

12. Élise Turcotte, Dans le delta de la nuit, Trois-Rivières, Écrits des Forges, 1982, 64 p.

13. Joseph Bonenfant, Grandes Aires, Trois-Rivières, Écrits des Forges, 1984, 68 p.

14. Carole Massé, Dieu, roman, Montréal, les Herbes rouges, 1979; et l'Existence, roman, les Herbes rouges, 1983.

15. Carole Massé, l'Autre, Montréal, revue les Herbes rouges, No 127, 1984, 46 p.

16. Carole Massé, "La femme à l'écritoire" in Qui a peur de l'écrivain? (en collaboration), revue les Herbes rouges, No 123-124, 1984, p. 64-83.

17. Roger des Roches, Poème, attention! suivi de Deuxième Poème, Montréal, revue les Herbes rouges, No 128, 1984, $40 \mathrm{p}$.

18. En fait pas du tout, la rédaction s'étend sur deux ans: «de juin 1982 à juin 1984».

19. Alexis Lefrançois, Comme tournant la page, poèmes 1968-1978, Saint-Lambert, le Noroît, 1984, $198 \mathrm{p}$.

20. Jean-Yves Collette, Préliminaires, textes 1965-1970, Saint-Lambert, le Noroît, 1984, 198 p.

21. Claude Beausoleil, les livres parlent, Trois-Rivières, Écrits des Forges, 1984, 246 p. 